\title{
Hepatitis C crónica: aspectos clínicos, serológicos y de tratamiento en dos centros de atención en Bogotá, Colombia
}

\section{Clinical Issues, Serological issues and Treatment of Chronic Hepatitis C at Two Medical Centers in Bogotá, Colombia}

\author{
Jhon E Prieto Ortiz, MD, ${ }^{1}$ Santiago Sánchez Pardo, MD, ${ }^{2}$ Leonardo Rojas Díaz, MD, ${ }^{3}$ Sandra Huertas Pacheco, MD. ${ }^{3}$
}

\author{
Gastroenterólogo Hepatólogo, Clínica Universitaria \\ Colombia. Bogotá, Colombia \\ 2 Médico Interno, Fundación Universitaria Sanitas. \\ Bogotá, Colombia \\ 3 Patóloga, Epidemióloga, Clínica Universitaria \\ Colombia. Bogotá, Colombia
}

Fecha recibido: $11-12-13$

Fecha aceptado: $05-11-14$

\begin{abstract}
Resumen
Introducción: el virus de la hepatitis C afecta a cerca de 170 millones de personas en el mundo. La organización mundial de la salud (OMS) estima una prevalencia mundial del $2 \%$. La respuesta global al tratamiento en la era de la terapia dual para genotipo 1 es del orden de 40\%. En Colombia hay datos limitados que confirmen un comportamiento similar y que describan las características clínicas de los pacientes con esta infección.

Metodología: se revisaron retrospectivamente las historias clínicas de pacientes con diagnóstico de hepatitis C crónica que asistieron a consulta externa del servicio de Hepatología en la Clínica Universitaria Colombia y de la consulta externa del servicio de Hepatología de uno de los autores durante el periodo comprendido entre el 1 de enero del 2010 y el 30 de mayo de 2013, se describen las características clínicas, serológicas y de respuesta al tratamiento.

Resultados: se evaluaron las historias clínicas de 163 pacientes, 62\% mujeres y 38\% hombres, con una edad promedio de 58,2 años. El principal factor de riesgo para la adquisición de la hepatitis $\mathrm{C}$ fue historia de transfusiones antes de 1992 en 62\% de los pacientes. La decisión de iniciar tratamiento se tomó en 77 pacientes $(47,2 \%)$ y en $86(52,8 \%)$ no se inició por diferentes razones dentro de las cuales la edad avanzada y cirrosis avanzada suman más de 50\%; otras razones para no iniciar el tratamiento fueron: enfermedad mínima (4,7\%), enfermedad mínima más edad avanzada (10,5\%), curación espontánea (14\%), poca probabilidad de respuesta $(3,3 \%)$ y otras (14\%). De 62 pacientes de los que se contaba con información acerca de tratamientos previos o tratados recientemente 30,6\% presentaron respuesta viral sostenida (RVS), 29,0\% fueron clasificados como reincidentes o relapser, 8,1\% como respondedores parciales, 19,4\% no tuvieron respuesta y $12,9 \%$ suspendieron el tratamiento por intolerancia.

Conclusiones: el antecedente más frecuente para la adquisición del VHC en el grupo de pacientes estudiado fue la historia de transfusiones antes de 1992 asociada con cirugía ginecológica. Cerca de la mitad de los pacientes se diagnostican tardíamente. Se muestra una mayor tendencia al tratamiento de la hepatitis con tasas de RVS similares a las encontradas en otras series. Este estudio abre puertas a la realización de otros que permitan definir de forma más amplia la prevalencia, factores de riesgo y variables de respuesta al tratamiento de esta entidad en nuestro país.
\end{abstract}

\section{Palabras clave}

Gastroenterología, virus de la hepatitis C, hepatitis C crónica, factores de riesgo, tratamiento. 


\section{Abstract}

Introduction: Hepatitis C affects about 170 million people worldwide. The World Health Organization (WHO) has estimated global prevalence at $2 \%$. Overall, about $40 \%$ of patients respond to dual therapy treatment for genotype. In Colombia data available for confirm a similar pattern and for describing the clinical characteristics of patients with this infection are scarce.

Methods: Medical records of patients in the Hepatology outpatient service at the Clínica Universitaria Colombia who had been diagnosed with chronic hepatitis $C$ by one of the authors between January 1, 2010 and May 30, 2013 were retrospectively reviewed for clinical characteristics, serological characteristics and treatment responses.

Results: The medical records of 163 patients were evaluated: $62 \%$ were female, $38 \%$ were male, and their mean age was 58.2 years. The main risk factor for acquiring hepatitis $\mathrm{C}$ was a history of transfusions before 1992. This factor was present in $62 \%$ of the patients. The decision to start treatment was made for 77 patients (47.2\%), but 86 patients (52.8\%) did not start treatment. Reasons included advanced age and advanced cirrhosis which together accounted for more than $50 \%$ of these patients. Other reasons for not starting treatment were minimal disease (4.7\%), minimal sign of disease plus advanced age (10.5\%), spontaneous healing (14\%), low probability of response (3.3\%) and others (14\%). Of the 62 patients for whom information about previous or recent treatments was available, 30.6\% had sustained virological responses (SVR), $29.0 \%$ were classified as relapsers, $8.1 \%$ as partial responders, $19.4 \%$ had no response, and $12.9 \%$ discontinued treatment because of intolerance.

Conclusions: The most frequent antecedent of HCV in the group of patients studied a history of transfusions associated with gynecological surgery before 1992. About half of the patients were diagnosed late. Hepatitis was more likely to have been treated in these patients than in patients in other studies, but the SVR rate was similar to those found in other series. This study opens doors to the realization of other studies to more broadly define the prevalence, risk factors and treatment response variables of this entity in our country.

\section{Keywords}

Gastroenterology, hepatitis C virus, hepatitis C infection, risk factors, treatment.

\section{MARCO TEÓRICO}

\section{Epidemiología}

Los virus de la hepatitis C (VHC) y B (VHB), son los causantes de la mayoría de hepatitis crónicas en el mundo, y una tercera parte de la población mundial ha estado en contacto con estos virus $(1,2)$. Se calcula en 130-170 millones el número de portadores del VHC, siendo la primera causa de cirrosis y trasplante hepático en países desarrollados y responsable de 1,2 millones de muertes al año debido a las complicaciones de la hipertensión portal secundaria a cirrosis: hemorragia de vías digestivas por várices esofágicas, ascitis, encefalopatía y carcinoma hepatocelular (3-6).

La organización mundial de la salud (OMS) estima una prevalencia de hepatitis $\mathrm{C}$ del orden de $2 \%$ de la población mundial, la mayoría en Asia (92 millones) y África (28 millones), en Europa se estima que puede haber unos 9 millones de afectados y en América unos 12 millones (2).

Para Estados Unidos datos del CDC de Atlanta (Centers for Disease Control) evidencian una caída en la incidencia de los casos de hepatitis $C$ aguda de 230.000 por año en los años 80 a 19.000 casos por año en registros recientes, con una incidencia actual de 0,3 por 100.000 habitantes y una prevalencia de 1 a 1,9\% (6-8). Para este mismo país la prevalencia de infección entre los años 1999 y 2002 fue de 1,6\%, lo que equivale a unos 4,1 millones de personas con anticuerpos contra el virus (anti-VHC) y de ellos $80 \%$ virémicos (8).

Para América Latina los datos varían de acuerdo a cada país de la región con una prevalencia en general de 1-2\%. Para Colombia, en el segundo trimestre de 2011 se reportaron 23 casos de hepatitis $\mathrm{C}$, calculando una incidencia de 0,5 X 100.000 habitantes, con una prevalencia $0,97 \%(9,10)$.

\section{Virus de la hepatitis C}

El virus de la hepatitis $C$ es un virus ARN y es el único representante del género Hepacivirus de la familia Flaviviridae, tiene un diámetro de aproximadamente $60 \mathrm{~nm}$ y solo infecta a los humanos y a los chimpancés; el virus se une a la superficie del hepatocito y entra a la célula por endocitosis. El RNA viral (con aproximadamente 9600 nucleótidos) codifica un precursor poliprotéico de aproximadamente 3000 aminoácidos; el reconocimiento citosólico de los productos virales induce la producción de citocinas proinflamatorias como el interferón llevando al recluta- 
miento de complejos de señalización para activar factores de transcripción. La expresión posterior de interferón- $\beta$ y el factor regulador del interferón 3 (IRF-3), inducen la respuesta inmune innata y la maduración del sistema inmune adaptativo para el control de la infección (11, 12). Se han descrito más de 6 genotipos y mas de 100 subtipos, pero aproximadamente $60-80 \%$ de las infecciones son causadas por el genotipo 1 (subtipos a y b) y 2 , otros genotipos son comunes en áreas como Egipto (genotipo 4), Suráfrica (genotipo 5) y el sur de Asia (genotipo 6) $(10,13)$. La determinación del genotipo es útil en estudios epidemiológicos y en el manejo clínico para predecir la probabilidad de respuesta al tratamiento y la duración óptima del mismo $(24,25)$. En Colombia según varios estudios el genotipo más frecuente es el 1 , en el estudio de Arias et al publicado en el año 2010 se encontró en $95 \%$ de 284 pacientes analizados (14-16).

\section{Factores de riesgo}

Los principales factores de riesgo descritos en la literatura internacional para adquirir la infección son el uso de drogas intravenosas, transfusiones sanguíneas antes de 1992, pacientes hemofílicos con transfusión de productos sanguíneos antes de 1987 y hemodiálisis, la transmisión maternofetal es muy rara y está asociada a la coinfección con VIH-1 (8), otros factores son el bajo nivel socioeconómico, un elevado número de parejas sexuales $(>20)(8,10,41)$, accidentes laborales en profesionales de la salud, los tatuajes, procedimientos odontológicos y endoscópicos (8-10).

\section{Historia natural de la infección}

La mayoría de su curso y la progresión a cirrosis transcurren en forma asintomática y sus manifestaciones solo se dan cuando el hígado es realmente insuficiente $(17,18)$. La infección aguda por el (VHC) representa aproximadamente $15 \%$ de los casos, de ellos solamente $25-30 \%$ de los pacientes son sintomáticos, con manifestaciones típicas de cualquier hepatitis viral, la hepatitis fulminante es excepcional $(10,19)$. La cronicidad por el virus de la hepatitis $\mathrm{C}$ se presenta hasta en $85 \%$ de los pacientes que adquieren la infección, 5-25\% desarrollan carcinoma hepatocelular después de ser portadores por más de 20 años y solamente $15-35 \%$ de los pacientes se curan espontáneamente del virus en los 6 meses siguientes a la primoinfección $(3,6,7$, 41). El progreso de la enfermedad hacia fibrosis o cirrosis esta relacionado con factores tales como: la edad en la que se adquiera la infección (antes o después de los 40 años), tiempo de duración de la infección (más de 20 años), sexo masculino, consumo de alcohol >50 gr/día, coinfección con otros virus como hepatitis B o VIH, la fuente de conta- gio, el estado de inmunocompetencia del huésped, factores propios del virus como el genotipo y la carga viral $(20,21)$.

\section{Tratamiento}

Estudios clásicos como los de Manns y Fried demostraron la utilidad del tratamiento en pacientes con hepatitis $\mathrm{C}$ crónica al lograr una respuesta viral sostenida (RVS) definida como niveles indetectables de RNA viral en sangre 6 meses después de terminado el tratamiento, con duración de 48 semanas para el genotipo 1 o 24 semanas para el genotipo 2, con respuesta promedio de 40 y $80 \%$ respectivamente, RVS que depende además del genotipo, del nivel de carga viral, grado de fibrosis, las características de la población y la adherencia al tratamiento (22-26). El tratamiento para la infección crónica hasta el año 2011 fue la combinación de interferón pegilado más ribavirina para ambos genotipos (47), en la actualidad y solamente para genotipo 1 , con el uso de los nuevos inhibidores de proteasa como el boceprevir y el telaprevir en conjunto con la terapia tradicional y la terapia guiada por respuesta (TGR) se ha logrado acortar la duración del tratamiento e incrementar la respuesta a este logrando tasas de RVS cercanas a $75 \%(27-33,41,47)$. Los pacientes relapsers o recaedores (pacientes con carga viral negativa al final del tratamiento que se hace positiva en los 6 meses siguientes de seguimiento), tienen tasas de RVS de hasta $80 \%$ en los retratamientos, siendo este grupo el de mayor indicación por encima de los respondedores parciales (pacientes con caída de más de 2 log a la semana 12 pero carga viral positiva a las semana 24 ), con RVS aproximada de $50 \%$ y respondedores nulos (caída menor de 2 log a la semana 12), con RVS aproximada de $30 \%(28,29,40,41)$. Otros blancos terapéuticos prometedores incluyen inhibidores de proteínas virales como core, proteína NS4B de la entrada viral, blancos del huésped como la ciclofilina A y la proteína miR122 (34) y recientemente dos nuevos medicamentos, sofosbuvir y simeprevir, fueron aprobados por los EE.UU. por la FDA para el tratamiento de la hepatitis C crónica (35).

\section{Biopsia hepática}

Necesaria en personas infectadas con el genotipo 1, puesto que el grado de fibrosis predice respuesta, define pronóstico y ayuda a concretar tratamiento en pacientes sin cirrosis (36-38), puede ser innecesaria en personas con infección por genotipos 2 y 3 del VHC, ya que más de $80 \%$ alcanzan una $\operatorname{RVS}(36,38-45)$.

\section{Justificación}

En nuestro medio no conocemos estudios publicados con información clínica y de tratamiento de pacientes con 
hepatitis $\mathrm{C}$ crónica, por tanto pretendemos realizar una aproximación acerca de la frecuencia de factores de riesgo ya establecidos y de las características clínicas y de tratamiento de un grupo de estos pacientes.

\section{Objetivo}

Describir características clínicas, serológicas y de respuesta al tratamiento en 163 pacientes adultos con diagnóstico de hepatitis $\mathrm{C}$ crónica que consultaron al servicio de hepatología de la Clínica Universitaria Colombia y a un servicio de consulta externa de hepatología en Bogotá durante el periodo del estudio.

\section{Metodología}

Se realizó la revisión retrospectiva de historias clínicas de los pacientes con hepatitis $\mathrm{C}$ crónica que fueron diagnosticados en la consulta o que ya tenían diagnóstico de la entidad y que asistieron a control en consulta externa del servicio de hepatología de la Clínica Universitaria Colombia y consulta externa del servicio de hepatología de uno de los autores durante el periodo comprendido entre el 1 de enero del 2010 y el 30 de mayo de 2013, posteriormente se tabularon los datos, se realizaron estadísticas descriptivas (Excel y SPSS) y se expresaron en forma de texto, tablas y gráficas o figuras.

\section{Diseño, población y definición de variables}

Se trata de un estudio descriptivo (clínico) retrospectivo en el cual se revisaron las historias clínicas de pacientes adultos con diagnóstico de hepatitis $\mathrm{C}$ crónica que asistieron a las consultas externas anotadas, se incluyeron únicamente aquellos pacientes en los que se conocía por lo menos un dato de carga viral positiva o habían sido tratados para hepatitis $\mathrm{C}$, se determinaron características demográficas (género, edad), motivo de remisión del paciente para la evaluación por hepatología, factores de riesgo para la infección por el virus de la hepatitis $\mathrm{C}$, datos del examen físico y pruebas de laboratorio incluyendo el genotipo y subtipo viral en los pacientes en que se contaba con este dato, adicionalmente se revisaron aspectos relacionados con tratamiento, clasificando a los pacientes según las características que los hacían candidatos o no para este; en los pacientes tratados previamente se revisó el tratamiento recibido y la respuesta, manejos adicionales de efectos adversos (de conocerse estos datos), en los pacientes que contaban con biopsia hepática se registró el estadio de la enfermedad utilizando la clasificación de Metavir (tablas 1 y 2 ).
Tabla 1. Distribución de los pacientes con diagnóstico de hepatitis C crónica según las variables demográficas, el motivo de remisión para consulta por hepatología y los factores de riesgo descritos.

\begin{tabular}{lcc}
\hline \multicolumn{1}{c}{ Variable } & N & $\%$ \\
\hline Género & \multicolumn{2}{c}{} \\
\hline Masculino & 62 & $38,0 \%$ \\
Femenino & 101 & $62,0 \%$ \\
Evaluación de hepatopatía (primera vez) & 75 & $46 \%$ \\
Control hepatitis C & 88 & $54 \%$ \\
\hline Motivo de remisión para consulta por hepatología(primera vez) \\
\hline Elevación de transaminasas & 35 & $46,6 \%$ \\
Cirrosis & 19 & $25,3 \%$ \\
Donación de sangre con Ac positivos para hepatitis C & 9 & $12 \%$ \\
Otros signos de hipertensión portal (ictericia, & 12 & $16 \%$ \\
sangrado varicial, otros) & \multicolumn{3}{|c}{} \\
\hline Factor de riesgo & 101 & $62,0 \%$ \\
\hline Transfusión & 6 & $3,7 \%$ \\
Hemofilia & 3 & $1,8 \%$ \\
Tatuajes & 2 & $1,2 \%$ \\
Acupuntura & 3 & $1,8 \%$ \\
Drogadicción & 40 & $24,5 \%$ \\
\hline Sin forma clara de adquisición
\end{tabular}

Tabla 2. Paraclínicos basales de los pacientes con hepatitis C crónica.

\begin{tabular}{|c|c|c|c|c|}
\hline \multicolumn{5}{|c|}{ Paraclínicos } \\
\hline Variable & Media & Mediana & Mínimo & Máximo \\
\hline $\begin{array}{l}\text { Recuento total de } \\
\text { leucocitos cel/ml }\end{array}$ & 5610,53 & 5400,00 & 1540 & 12900 \\
\hline Hemoglobina g/dl & 14,45 & 15,00 & 7 & 19 \\
\hline Hematocrito \% & 43,90 & 45,00 & 22 & 57 \\
\hline $\begin{array}{l}\text { Recuento plaquetario } \\
\text { cel/ml }\end{array}$ & 207225,17 & 208000,00 & 27000 & 444000 \\
\hline Glicemia mg/dl & 97,04 & 93,00 & 70 & 188 \\
\hline Nitrógeno ureico mg/dl & 17,41 & 15,00 & 6 & 88 \\
\hline Creatinina mg/dl & 856 & 800 & ,2 & 4,6 \\
\hline Colesterol total mg/dl & 176,88 & 174,00 & 81 & 330 \\
\hline TSH uUl/ml & 3,114 & 2,500 & 1 & 16,0 \\
\hline \multicolumn{5}{|c|}{ Función hepática } \\
\hline AST UI/ml & 68,32 & 48,50 & 15 & 302 \\
\hline ALT Ul/ml & 80,48 & 54,00 & 9 & 461 \\
\hline GGT U/l & 86,97 & 57,00 & 8 & 719 \\
\hline Fosfatasa alcalina U/ml & 117,55 & 96,00 & 3 & 810 \\
\hline TP paciente seg & 12,43 & 12,00 & 9 & 25 \\
\hline INR & 1,079 & 1,000 & ,8 & 2,5 \\
\hline Bilirrubina total mg/dl & 1,015 &, 700 & 1 & 9,7 \\
\hline Bilirrubina directa mg/dl &, 369 & ,200 & 0,0 & 5,3 \\
\hline Bilirrubina indirecta mg/dl & ,624 &, 500 & 0,0 & 4,4 \\
\hline Proteínas totales gr/dl & 7,344 & 7,400 & 6,0 & 9,0 \\
\hline Albúmina gr/dl & 4,062 & 4,100 & 2,1 & 5,0 \\
\hline Carga viral UI/I & 803683,09 & 247719,00 & 0 & 5812593 \\
\hline
\end{tabular}




\section{Análisis estadístico}

Se realizó análisis descriptivo de cada una de las variables a estudio. Para las variables continuas se estimaron promedios y desviaciones estándar, para las variables categóricas se estimaron proporciones. Se analizó la información mediante estadísticas descriptivas (medidas de frecuencia) y se presentaron en texto, tablas y/o gráficas, empleando EXCEL y SPSS.

\section{Consideraciones éticas}

Este es un estudio retrospectivo que utilizó datos de las historias clínicas de pacientes; por lo tanto se considera "sin riesgo" de acuerdo con la clasificación establecida en la resolución 8430 del Ministerio de Salud de 1993, teniendo en cuenta que no se realizó ninguna intervención o modificación intencionada de variables biológicas, fisiológicas, psicológicas o sociales de los individuos participantes. El estudio se condujo de acuerdo con los principios declarados en la XVIII Asamblea Médica Mundial (Helsinki, 1964). Los datos de identificación de los pacientes incluidos así como sus diagnósticos no fueron consignados dentro de las publicaciones y el grupo de investigadores manejó con total confidencialidad los datos de historia clínica.

\section{RESULTADOS}

\section{Características generales de los pacientes}

Del total de los pacientes 101 (62\%) eran mujeres y 62 (38\%) hombres, con una edad promedio de 58,2 años (21-94 años) (figura 1), 75 pacientes (46\%) asistieron por primera vez y 88 (54\%) eran controles de hepatitis C; dentro de los pacientes que asistían por primera vez a la consulta, los principales motivos de remisión a hepatología fueron: transaminasas altas en $35(46,7 \%)$, cirrosis en 19 pacientes (25,3\%), donación de sangre con anticuerpos de hepatitis $\mathrm{C}$ positivos en 9 (12\%), otros signos de HTP en 12 (16\%). Dentro de los factores de riesgo para la adquisición de la hepatitis $\mathrm{C}$ el más frecuente en nuestra serie fue el antecedente de transfusiones sanguíneas antes de 1992 demostrado en 101 pacientes que representan $62 \%$. Las causas de dichas transfusiones fueron: cirugía ginecológica en 45 pacientes $(44,6 \%)$, cirugía relacionada con trauma en 12 pacientes $(11,9 \%)$, cirugía ortopédica en $9(8,9 \%)$, cirugía de úlcera gastrointestinal en 11 (10,9\%), otros tipos de cirugía en 24 (23,8\% incluyendo cirugía cardiovasculares, de amígdalas entre otras). Otros factores de riesgo encontrados fueron hemofilia, tatuajes, acupuntura y drogadicción en $3,7 \%, 1,8 \%, 1,2 \%$ y $1,8 \%$ respectivamente.

\section{Examen físico y estratificación del grado de enfermedad}

Del total de los pacientes, $61(37,4 \%)$ presentaban estigmas de hepatopatía crónica y $102(62,6 \%)$ tenían un examen físico normal. De los 61 pacientes con un examen físico anormal se encontró ictericia en $6(3,7 \%)$, ascitis en 27 $(16,6 \%)$, hígado con consistencia aumentada o sugestiva de cirrosis en 56 (34,4\%), bazo palpable en 26 (16\%) y nevus arácneos en 27 (16,6\%). El diagnóstico de cirrosis se estableció en 52 pacientes $(31,9 \%)(n=163)$, el CHILD se determinó en 35 de ellos, de los cuales 21 se clasificaron como CHILD A (60\%), B 13 pacientes $(37,1 \%)$ y C 1 $(2,9 \%)$ como se muestra en la figura 2 . La biopsia hepática se realizó en 71 pacientes $(43,5 \%)$ y de ellos $10(14,1 \%)$ se encontraban en cirrosis con estadio F4 (Metavir), las demás distribuciones se muestran en la figura 3.

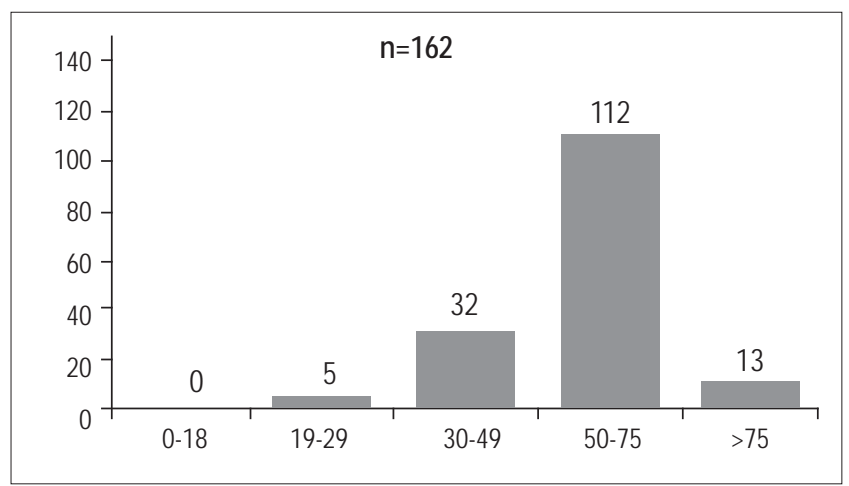

Figura 1. Distribución por edades de los pacientes con hepatitis C crónica.

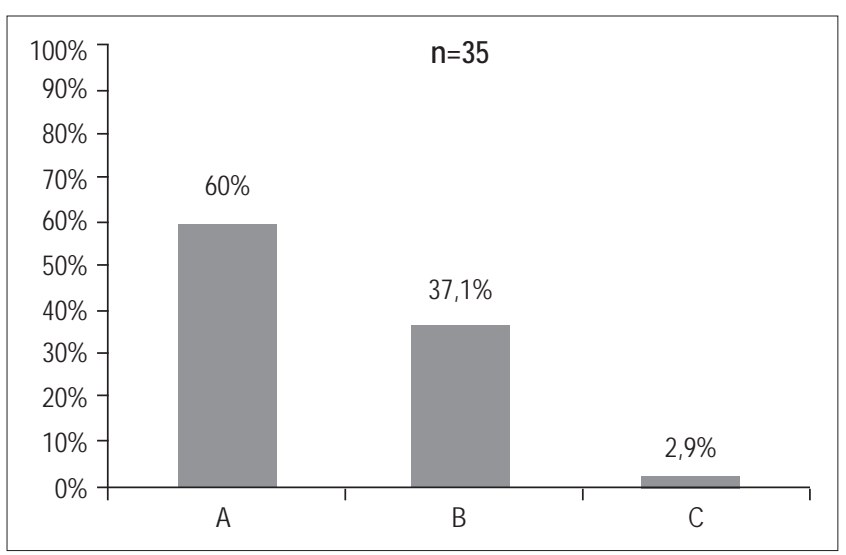

Figura 2. Valoración clínica de los pacientes utilizando la puntuación de CHILD.

\section{Aspectos del virus de la hepatitis C}

El análisis del genotipo viral se hizo en 56 pacientes, siendo los dos más frecuentes el genotipo $1 \mathrm{~B}$ en 44 pacientes $(78,5 \%)$ y el $1 \mathrm{~A}$ en 8 pacientes (14,2\%), seguidos de los genotipos $2 \mathrm{~B}, 1 \mathrm{~A}-1 \mathrm{~B}$, IV y 1 sin subtipificacion con $1,7 \%$ para cada uno de ellos. El promedio de la carga viral fue de 803683 de UI / $\mathrm{ml}$ con una mediana de 247719 UI / ml. 


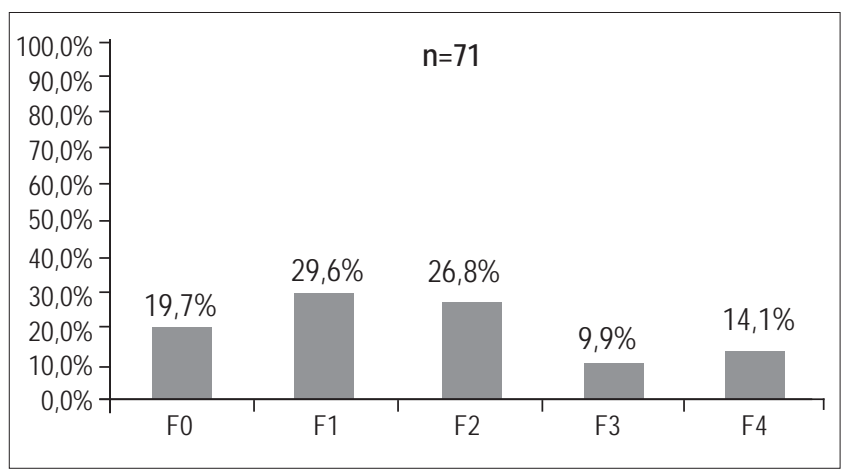

Figura 3. Estadio de la lesión hepática determinado por biopsia (Metavir).

\section{Aspectos relacionados con el tratamiento}

En un primer análisis en 84 pacientes con opción de tratamiento o tratados, se revisó el número de tratamientos encontrando los siguientes subgrupos; 23 no habían recibido ningún tratamiento, es decir eran pacientes "naives" $(27,3 \%), 43(51,2 \%)$ habían recibido un tratamiento previo, $13(15,5 \%) 2$ tratamientos, $4(4,8 \%)$ tres y 1 paciente $(1,2 \%) 4$ o más. En 62 pacientes tratados en el pasado o recientemente se contó con datos de respuesta al tratamiento de los cuales $19(30,6 \%)$ presentaron respuesta viral sostenida (RVS), $18(29,0 \%)$ recayeron luego del tratamiento (relapsers), 5 (8,1\%) fueron respondedores parciales, $12(19,4 \%)$ no tuvieron respuesta y $8(12,9 \%)$ suspendieron el tratamiento por intolerancia. En un segundo análisis se toman todos los pacientes que se trataron o que tienen nuevas indicaciones de tratamiento (pacientes naives, relapsers, respondedores parciales, se incluyen 14 pacientes con terapia triple con boceprevir y/o telaprevir), representan el grupo de 77 pacientes que se muestran en la figura 4. De los 163 pacientes del estudio, no se considero indicado el tratamiento para el VHC en $86(52,8 \%)$ por diferentes razones: Enfermedad mínima 4 pacientes $(4,7 \%)$, enfermedad mínima mas edad avanzada $9(10,5 \%)$, edad avanzada $15(17,4 \%)$, cirrosis avanzada 29 $(33,7 \%)$ y curación espontánea $12(14 \%)$, poca probabilidad de respuesta $3(3,3 \%)$ y otras en $14(16,3 \%)$. En 77 pacientes se consideró tratamiento y los resultados fueron: en evaluación 31 (40,3\%), en espera de iniciarlo 7 (9,1\%), en tratamiento al momento del estudio $11(14,3 \%)$, tratados y curados en el punto de corte $19(24,7 \%)$, tratados no curados $9(11,7 \%)$ (figuras 4 y 5$)$.

\section{Estadio clínico de la hepatitis C}

En 162 pacientes analizando los datos clínicos, de laboratorio, estudio de patología y la información de respuesta al tratamiento se pudo establecer el estadio clínico de la enfer- medad con la siguiente distribución: $41(25,3 \%)$ presentaban cirrosis, 11 cirrosis más hepatocarcinoma (6,8\%), 80 hepatitis C crónica $(49,4 \%)$ y 30 hepatitis C curada ya sea por curación espontánea o por tratamiento $(18,5 \%)$, concomitantemente se presentó hígado graso en 54 pacientes $(34,8 \%)$, diagnosticados por biopsia y ecografía, de ellos $39(72,2 \%)$ presentaban NAFLD (del inglés Non-alcoholic fatty liver disease) y $15(27,8 \%)$ NASH (del inglés nonalcoholic steatohepatitis) (figura 6).

\begin{tabular}{|c|c|}
\hline \multicolumn{2}{|c|}{$\begin{array}{l}\text { Aspectos relacionados con el tratamiento } \\
\qquad \mathrm{N}=163(100 \%)\end{array}$} \\
\hline$\nabla$ & $\nabla$ \\
\hline $\begin{array}{l}\text { No se consideró el tratamiento } \\
\qquad \mathrm{N}=86(52,8 \%)\end{array}$ & $\begin{array}{l}\text { Si se consideró el tratamiento } \\
\qquad \mathrm{N}=77(47,2 \%)\end{array}$ \\
\hline$\downarrow$ & $\downarrow$ \\
\hline 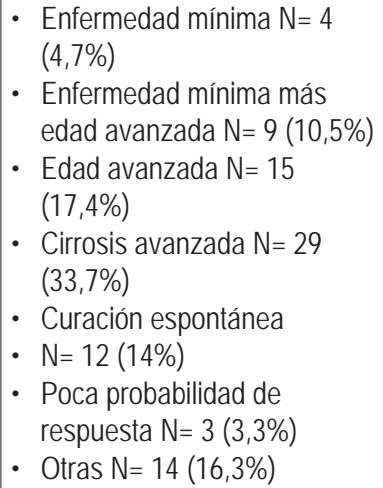 & 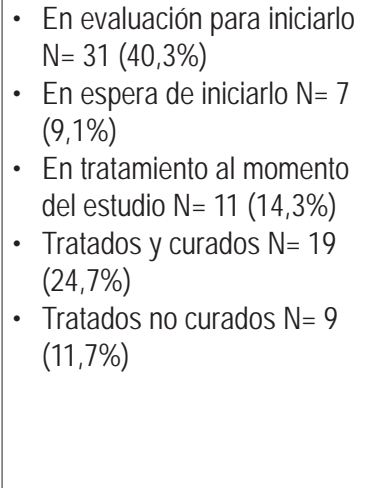 \\
\hline
\end{tabular}

Figura 4. Características de los pacientes con respecto al tratamiento.

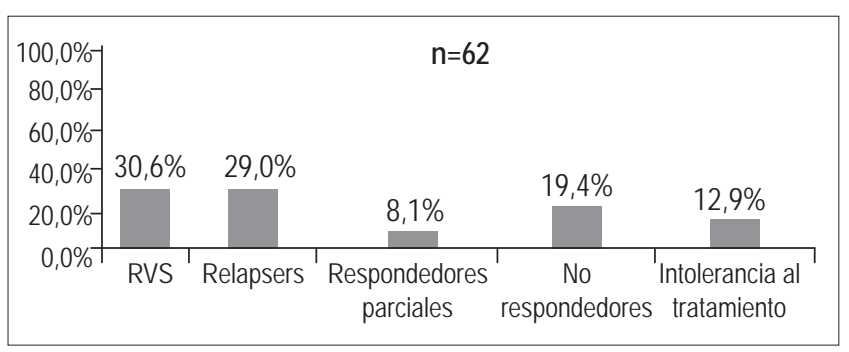

Figura 5. Tipos de respuesta al tratamiento.

\section{DISCUSIÓN}

El mayor porcentaje de pacientes corresponde al género femenino (62\%) y la edad promedio del total de los pacientes fue de 58,2 años, esto se explica porque en nuestra serie el factor de riesgo más frecuentemente identificado para la adquisición de la hepatitis $\mathrm{C}$ fue el antecedente de una 


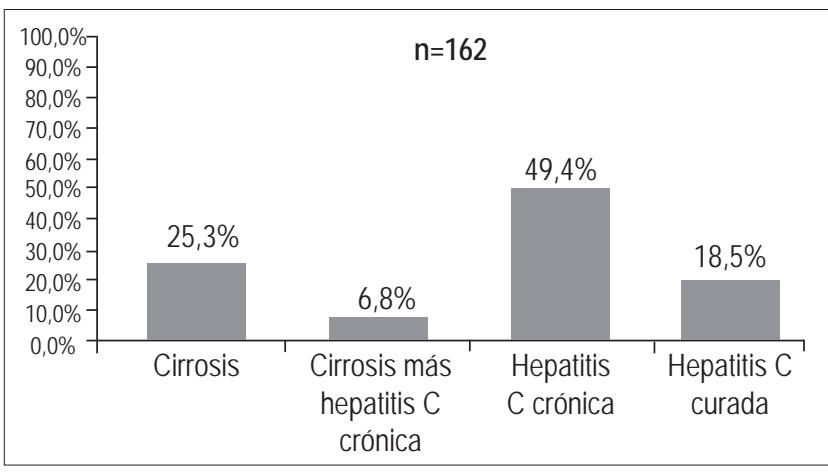

Figura 6. Estadio clínico de la hepatitis C.

transfusión antes de 1992 (en 101 pacientes, 62\%), en su mayoría mujeres que fueron transfundidas por cirugías relacionadas con la gestación hace 20 o 30 años. La distribución bimodal descrita en otras series (41), asociada al uso de drogas intravenosas entre las personas de 30-49 años no se observó ya que la drogadicción en nuestra serie fue baja, cercana a $2 \%$. En 40 pacientes $(24,5 \%)$ la forma de adquisición no se pudo determinar, en estudios internacionales como el de McCarthy estos porcentajes están alrededor de $40 \%$ (46), creemos que estos datos se deben a una mayor trasmisión postransfusional en nuestro medio, 5 pacientes que representan 3,1\% eran trabajadores de la salud, lo cual llama la atención e invita al fortalecimiento en las medidas de bioseguridad intrahospitalarias (8-10). En relación con el virus de la hepatitis $C$, se encontró al igual que en el estudio previo en nuestro medio (14-16), el genotipo 1 como el predominante en $96,4 \%$ de los pacientes, con un promedio de la carga viral fue de $803.683 \mathrm{UI} / \mathrm{ml}$ dato importante, ya que se conoce que hay mayor fibrosis a mayor carga viral (valores mayores de $800.000 \mathrm{UI} / \mathrm{ml}$ ) como se demostró en el estudio de Hadziyanis (36) y el genotipo 1 implica mayor dificultad en el tratamiento con menores tasas de $\operatorname{RVS}(15,47,49)$.

Datos de la biopsia hepática se obtuvieron en 71 pacientes, la mayoría de ellas dentro del grupo de pacientes en que se consideró tratamiento, por seguimiento de los estándares internacionales que la recomiendan para inicio de este $(38,42-44,48,49)$.

Se consideró tratamiento en nuestra serie en $47 \%$ de los pacientes, lo iniciaron 46 (28,2\%), con RVS en 19 pacientes que corresponden a $67 \%$ de los que se tratan, $24 \%$ de los que se consideran para tratamiento y en $11 \%$ de todos los pacientes. Los estudios clásicos de Mans, Fried y Mchutchinson en pacientes tratados, muestran tasas de RVS de 42,46 y $40 \%$ respectivamente $(22,23,53)$; en el estudio de Butt, en una serie de 134.934 veteranos infectados con el VHC solamente $11 \%$ inician el tratamiento y de ellos solo $22 \%$ terminan las 48 semanas $(50,51)$. Los datos que encontramos en nuestra serie no son comparables con los internacionales, pero nos muestran, aunque en forma inicial, cuál es el comportamiento de nuestros pacientes y nos induce a ampliar el número de pacientes de la serie.

En el grupo de pacientes en quienes no se consideró tratamiento (53\%), llama la atención que en conjunto edad avanzada $15(17,4 \%)$ y cirrosis avanzada $29(33,7 \%)$ suman más de $50 \%$ del total, grupo que se hubiera beneficiado de una detección más precoz de la infección, en un momento con mayor posibilidad de tratamiento. En nuestra serie el grupo de curación espontánea $14 \%$ contrasta con $25-35 \%$ descrito en la literatura internacional (52-56), la única asociación que podemos establecer, es la presencia del genotipo uno en más de $90 \%$ de nuestros pacientes.

Con respecto al diagnóstico de la hepatopatía, se resalta que en casi la mitad de los pacientes, 80 (49\%), se definió el estadio de la hepatopatía como hepatitis C crónica, condición en la cual por definición se puede considerar tratamiento (26-28). Se diagnosticó cirrosis hepática en 52 pacientes $(31,9 \%)$ (incluidos 11 con cirrosis más hepatocarcinoma), este grupo en algún momento de la historia natural podría ser candidato a trasplante hepático, sabemos que en EE.UU. la hepatitis $C$ es la principal indicación en la actualidad de este procedimiento y esto, trasladado a nuestro país, es un problema de proporciones gigantescas, teniendo en cuenta que basados en una probable prevalencia de la enfermedad en nuestro medio de $1 \%$, es decir, aproximadamente 500.000 casos de hepatitis $\mathrm{C}$, que todavía no hemos diagnosticado (10).

Este estudio describe características clínicas, serológicas y de respuesta al tratamiento de los pacientes con diagnóstico de hepatitis $\mathrm{C}$ crónica con terapia dual fundamentalmente, aunque incluye datos parciales de pacientes con terapia triple y deja clara la necesidad de ampliar la serie para consolidar datos de factores de riesgo y respuesta al tratamiento de esta entidad en nuestro medio, así mismo se invita a plantear series de prevalencia mediante estudios poblacionales para conocer la verdadera importancia de la $\mathrm{HCV}$ en Colombia.

\section{REFERENCIAS}

1. Armstrong GL, Wasley A, Simard EP, McQuillan GM, Kuhnert WL, Alter MJ. The prevalence of hepatitis C virus infection in the United States, 1999 through 2002. Ann Intern Med 2006; 144: 705-14.

2. Averhoff FM, Glass N, Holtzman D, Global burden of hepatitis C: considerations for healthcare providers in the United States, Clin Infect Di 2012; 55(Suppl. 1): S10-5.

3. Louie K, St Laurent S, Forssen U, Mundy L, Pimenta J. The high comorbidity burden of the hepatitis $\mathrm{C}$ virus infected population in the United States. BMC Infectious Diseases 2012; 1286. 
4. Burguete-García A, Conde-González C, Jiménez-Méndez R, Juárez-Díaz Y, Meda-Monzón E, Madrid-Marina V, et al. Hepatitis $\mathrm{C}$ seroprevalence and correlation between viral load and viral genotype among primary care clients in Mexico. Salud Pública de México 2011; 53S7-S12.

5. Garcia-Tsao G, Sanyal AJ, Grace ND, Carey W; Practice Guidelines Committee of the American Association for the Study of Liver Diseases; Practice Parameters Committee of the American College of Gastroenterology. Prevention and management of gastroesophageal varices and variceal hemorrhage in cirrhosis. Hepatology 2007; 46: 922-38.

6. Lavanchi D. Evolving Epidemiology of Hepatitis C Virus. Clin Microbiol Infect 2011; 17: 107-115.

7. Aman W, Mousa S, Shiha G, Mousa S. Current status and future directions in the management of chronic hepatitis $\mathrm{C}$. Virology Journal 2012; 957.

8. Louie, et al. The high comorbidity burden of the hepatitis $\mathrm{C}$ virus infected population in the United States, BMC Infectious Diseases 2012; 12: 86.

9. Informe comportamiento de la hepatitis b y c II trimestre 2011 SIVIGILA II trimestre 2011.

10. Guías de diagnóstico y tratamiento de hepatitis C. Rev Col Gastroenterol 2012; 27 (Supl. 4).

11. Horner SM, Gale M Jr. Intracellular innate immune cascades and interferon defenses that control hepatitis $\mathrm{C}$ virus. J Interferon Cytokine Res 2009; 29: 489-98.

12. Thio CL, Thomas DL. Interleukin-28b: a key piece of the hepatitis C virus recovery puzzle. Gastroenterology 2010; 138: 1240-3.

13. Beltrán M, Navas MC, De la Hoz F, Mercedes Muñoz M, Jaramillo S, et al. Hepatitis C virus seroprevalence in multi-transfused patients in Colombia. J Clin Virol 2005; 34(Suppl. 2): S33-38.

14. Arias Y R, Echeverry S J, Castro M A, Rios M F, Martinez $\mathrm{O}$, frecuencia de genotipos y subtipos de virus de la hepatitis c en pacientes colombianos con infección crónica. Rev Medica Sanitas 2010; 13 (3): 10-19.

15. Farfan YA, Garzón MA, Rey MH. Prevalencia de hepatitis $\mathrm{C}$ por reacción de cadena de la polimerasa (PCR), en donantes de Banco de sangre. Revista Colombiana de Gastroenterología 2007; 22(4): 308-312.

16. Botero R, Idrovo V, et al. Genotipos de VHC. Revista Colombiana de Gastroenterología 1998; XII: 25-27.

17. Leonard B. Seeff, Natural History of Chronic Hepatitis C. Hepatology 2002; 36(5).

18. Oh R, Hustead T. Causes and evaluation of mildly elevated liver transaminase levels. American Family Physician 2011; 84(9): 1003-1008.

19. Seeff LB. Why is there such difficulty in defining the natural history of hepatitis? Transfusion 2000; 40: 1161-1164.

20. Paul H. Hayashi, Adrian M. Di Bisceglie, The progression of hepatitis $\mathrm{B}-$ and $\mathrm{C}$-infections to chronic liver disease and hepatocellular carcinoma: epidemiology and pathogenesis, Med Clin N Am 2005; 89 371-389.

21. Hugo R. Rosen, Chronic Hepatitis C Infection, N Engl J Med 2011; 364: 2429-38.
22. Manns MP, et al. Peginterferon alfa- $2 b$ plus ribavirin compared with interferon alfa- $2 \mathrm{~b}$ plus ribavirin for initial treatment of chronic hepatitis C: a randomised trial. Lancet 2001; 358: 958-965.

23. Fried MW, Shiffman ML, Reddy KR, et al. Peginterferon alfa-2a plus ribavirin for chronic hepatitis $\mathrm{C}$ virus infection. N Engl J Med 2002; 347: 975-82.

24. Simmonds P, Bukh J, Combet C, Deleage G, Enomoto N, Feinstone $S$, et al. Consensus proposals for a unified system of nomenclature of hepatitis $\mathrm{C}$ virus genotypes. Hepatology 2005; 42: 962-973.

25. Vanegas N, Román J, Febles M, Sánchez C, Suárez J, López $\mathrm{M}$, et al. Tratamiento de la infección crónica por el virus de la hepatitis C. Factores predictores de respuesta. Revista Española De Quimioterapia 2011; 24(4): 198-203.

26. Poordad F, McCone J Jr, Bacon BR, Bruno S, Manns MP, Sulkowski MS, et al; SPRINT-2 Investigators. Boceprevir for untreated chronic $\mathrm{HCV}$ genotype 1 infection. N Engl J Med 2011; 364: 1195-206.

27. Jacobson IM, McHutchison JG, Dusheiko G, Di Bisceglie AM, Reddy KR, Bzowej NH, et al; ADVANCE Study Team. Telaprevir for previously untreated chronic hepatitis $\mathrm{C}$ virus infection. N Engl J Med 2011; 364: 2405-16.

28. Zeuzem Stefan, Andreone Pietro, Pol Stanislas, Lawitz Eric, Diago Moises, Roberts Stuart, et al, Telaprevir for Retreatment of HCV Infection, N Engl J Med 2011; 364: 2417-28.

29. Bacon R. Bruce, Gordon C Stuart, Lawitz Eric, Marcellin Patrick, Vierling M John, Zeuzem Stefan, Boceprevir for Previously Treated Chronic HCV Genotype 1 Infection, N Engl J Med 2011; 364: 1207-17.

30. Shan Liu, Lauren E. Cipriano, Mark Holodniy, Douglas K. Owens, Jeremy D. Goldhaber-Fiebert, New Protease Inhibitors for the Treatment of Chronic Hepatitis C, A CostEffectiveness Analysis, Annals of Internal Medicine 2012; 156: 4.

31. Bezemer Geert et al. Long-term effects of treatment and response in patients with chronic hepatitis $\mathrm{C}$ on quality of life. An international, multicenter, randomized, controlled study, BMC Gastroenterology 2012; 12: 11.

32. Haider S, Ahmad J. Update of old and emerging therapies in chronic hepatitis C. JPMA. The Journal of The Pakistan Medical Association 2011; 61(12): 1226-1230.

33. Cisneros-Garza L. Nuevos avances en el manejo de la hepatitis C. Salud Pública de México 2011; 53S52-S60.

34. T. Jake Liang, Marc G. Ghany, Current and Future Therapies for Hepatitis C Virus Infection, N Engl J Med 2013; 368: 1907-17.

35. Chao D1, Botwin GJ, Morgan TR. Update on Recently Approved Treatments for Hepatitis C. Curr Treat Options Gastroenterol 2014. [Epub ahead of print].

36. Hadziyannis SJ, et al. Peginterferon-alpha2a and ribavirin combination therapy in chronic hepatitis $\mathrm{C}$ : a randomized study of treatment duration and ribavirin dose. Ann Intern Med 2004; 140: 346-355. 
37. Brunt EM. Grading and staging the histopathological lesions of chronic hepatitis: the Knodell histology activity index and beyond. Hepatology 2000; 31: 241-246.

38. Wong JB, Koff RS. Watchful waiting with periodic liver biopsy versus immediate empirical therapy for histologically mild chronic hepatitis C. A cost-effectiveness analysis. Ann Intern Med 2000; 133: 665-675.

39. Lee J, Payette M, Osiecki J. Viral hepatitis: targeted tests and therapies contribute to improved outcomes. MLO: Medical Laboratory Observer 2012; 44(3): 18-20.

40. Mach T, Cieśla A, Warunek W, Janas-Skulina U, Cibor D, Ciećko-Michalska I, et al. Efficacy of pegylated interferon alfa- $2 a$ or alfa- $2 b$ in combination with ribavirin in the treatment of chronic hepatitis caused by hepatitis $\mathrm{C}$ virus genotype 1b. Polskie Archiwum Medycyny Wewnętrznej 2011; 121(12): 434-439.

41. Ghany, et al. Diagnosis, Management, and Treatment of Hepatitis C: An Update. Hepatology 2009; 49(4).

42. Kleiner DE. The liver biopsy in chronic hepatitis C: a view from the other side of the microscope. Semin Liver Dis 2005; 25: 52-64.

43. Gebo A Kelly, et al. Role of Liver Biopsy in Management of Chronic Hepatitis C: A Systematic Review. Hepatology 2002; 36(5): 1 .

44. Ishak K, Baptista A, Bianchi L, Callea F, De Groote J, Gudat $\mathrm{F}$, et al. Histological grading and staging of chronic hepatitis. J Hepatol 1995; 22: 696-699.

45. Abraira García, Luisa, García Sierra, Alberto, Guillán Pavón, Begoña. Otero Antón, Esteban, Suárez López, Francisco. Guía de práctica clínica Hepatitis C. Santiago de Compostela 2009.

46. McCarthy M, Wilkinson ML. Hepatology. BMJ (Clinical Research Ed.) 2000; 318: 1256-9.

47. Ghany MG, Nelson DR, Strader DB, Thomas DL, Seeff LB. An update on treatment of genotype 1 chronic hepatitis $\mathrm{C}$ virus infection: 2011 practice guideline by the American
Association for the Study of Liver Diseases. Hepatology 2011; 54: 1433-I444.

48. Foster GR, Fried MW, Hadziyannis SJ, Messinger D, Freivogel K,Welland $\mathrm{O}$ Prediction of sustained virological response in chronic hepatitis $\mathrm{C}$ patients treated with peginterferon alfa $-2 \mathrm{a}$ and ribavirin. Scan J Gastroenterol 2007; 42(7): 247-255.

49. Valva P, Casciato P, Diaz Carrasco JM, Gadano A, Galdame $\mathrm{O}$, et al. The Role of Serum Biomarkers in Predicting Fibrosis Progression in Pediatric and Adult Hepatitis C Virus Chronic Infection, 2011 PLoS ONE 6(8): e23218.

50. Falck-Ytter Y, Kale H, Mullen KD, et al. Surprisingly small effect of antiviral treatment in patients with hepatitis C. Ann Intern Med 2002; 136: 288.

51. Butt AA, McGinnis KA, Skanderson M, Justice AC. Hepatitis $\mathrm{C}$ treatment completion rates in routine clinical care. Liver Int 2010; 30: 240.

52. Kanwal F, Hoang T, Spiegel BM, et al. Predictors of treatment in patients with chronic hepatitis $\mathrm{C}$ infection - role of patient versus nonpatient factors. Hepatology 2007; 46: 1741.

53. McHutchison G John, Lawitz J Eric, Shiffman Mitchell, Muir J Andrew, Galler W Greg, McCone Jonathan, Peginterferon Alfa-2b or Alfa-2a with Ribavirin for Treatment of Hepatitis C Infection, N Engl J Med 2009; 361: 580-93.

54. Barrett S, Goh J, Coughlan B, et al. The natural course of hepatitis $\mathrm{C}$ virus infection after 22 years in a unique homogenous cohort: spontaneous viral clearance and chronic HCV infection. Gut 2001; 49: 423.

55. Datz C, Cramp M, Haas T, et al. The natural course of hepatitis $\mathrm{C}$ virus infection 18 years after an epidemic outbreak of non-A, non-B hepatitis in a plasmapheresis centre. Gut 1999; 44: 563.

56. Seeff LB, Miller RN, Rabkin CS, et al. 45-year follow-up of hepatitis $\mathrm{C}$ virus infection in healthy young adults. Ann Intern Med 2000; 132: 10. 